\title{
ON DIFFERENTIAL EQUATIONS ADMITTING POLYGENIC INTEGRALS*
}

\author{
BY \\ GEORGES CALUGAREANO
}

We have shown, in a previous paper, $\dagger$ that some differential equations admit more general integrals than those given by the theorem of Cauchy; indeed, this theorem which uses majorant functions gives analytic integrals for each differential equation and we have seen that there are equations which admit polygenic integrals. But in the paper mentioned above we did not succeed in defining completely the class to which those equations belong and which we shall denote by class $(\alpha)$. We shall show that this class may be completely defined by a property of its general monogenic integral.

1. Let us consider the second-order equation.

$$
y^{\prime \prime}=F\left(z, y, y^{\prime}\right)
$$

which belongs to the class $(\alpha)$, admitting the polygenic integral

$$
y=\phi(z, \bar{z}),
$$

where $\phi$ is a monogenic function in respect to each of the variables $z$ and $\bar{z}$. If $\mu=e^{-2 i \theta}$ we have

$$
y^{\prime}=\phi_{z}+\mu \phi_{\bar{z}} ; y^{\prime \prime}=\phi_{2,}+2 \mu \phi_{z \bar{z}}+\mu^{2} \phi_{\bar{z}}
$$

and eliminating $\mu$ we get

$$
y=\phi_{z i}+2 \phi_{z \bar{z}} \frac{y^{\prime}-\phi_{z}}{\phi_{z}}+\phi_{\bar{z}^{2}}\left[\frac{y^{\prime}-\phi_{z}}{\phi_{\bar{z}}}\right]^{2} .
$$

Substituting in (4) the value of $\bar{z}$ deduced from (2) we obtain an equation of the following form:

$$
y^{\prime \prime}=A(z, y) \cdot y^{\prime 2}+B(z, y) \cdot y^{\prime}+C(z, y),
$$

hence

$$
F\left(z, y, y^{\prime}\right)=A(z, y) \cdot y^{\prime 2}+B(z, y) \cdot y^{\prime}+C(z, y),
$$

which holds good for any value of $\theta$, hence for any value of $y^{\prime}$ on the corre-

* Presented to the Society, December 27, 1929; received by the editors in September, 1929.

† See these Transactions, vol. 31, p. 372. 
sponding Kasner* circle. The expression $\left(5^{\prime}\right)$ is therefore an identity in $y^{\prime}$, and hence any equation of the second order belonging to class $(\alpha)$ is of form (5). We get then easily the supplementary conditions for the determination of $A, B, C$.

It is to be remarked that the elimination made above holds without any modification if we start from

$$
y=\phi\left(z, \gamma_{\mathrm{c}} \bar{z}+\gamma_{1} z+\gamma_{2}\right),
$$

where $\gamma_{0}, \gamma_{1}$ and $\gamma_{2}$ are arbitrary constants.

Indeed, if $k=\gamma_{0} \bar{z}+\gamma_{1} z+\gamma_{2}$ we have

(7) $y^{\prime}=\frac{\partial \phi}{\partial z}+\left(\gamma_{1}+\mu \gamma_{0}\right) \frac{\partial \phi}{\partial k} ; \cdots ; y^{(n)}=\left(\frac{\partial}{\partial z}+\left(\gamma_{1}+\mu \gamma_{0}\right) \frac{\partial}{\partial k}\right)^{(n)} \phi$,

and the elimination of $\gamma_{1}+\mu \gamma_{0}$ and $k$ between (6) and (7) is equivalent to the elimination of $\mu$ and $\bar{z}$ between (2) and (3). But (6) shows that $y$ has a special property. We know that the monogenic integral of (1) is obtained from (6) by replacing $\bar{z}$ by $z-C$. The same result is obtained if we make $\gamma_{0}=0$, and this shows that the monogenic integral of (1) is of the form

$$
Y=\phi\left(z, \gamma_{1} z+\gamma_{2}\right) \text {. }
$$

This integral depends in a special manner on the arbitrary constants $\gamma_{1}$ and $\gamma_{2}$. This property furnishes the characterization of the whole class $(\alpha)$.

2. Let

$$
y=\phi\left(z, \bar{z}, \gamma_{3}, \gamma_{4}, \cdots, \gamma_{n}\right)
$$

be a polygenic function depending on $n-2$ arbitrary constants, $\boldsymbol{\gamma}_{\boldsymbol{s}}, \boldsymbol{\gamma}_{\mathbf{4}}, \cdots$, $\gamma_{n}$. We can write

$$
y^{\prime}=\frac{\partial \phi}{\partial \bar{z}}+\mu \frac{\partial \phi}{\partial \bar{z}} ; \cdots ; y^{(n)}=\left(\frac{\partial}{\partial z}+\mu \frac{\partial}{\partial \bar{z}}\right)^{(n)} \phi,
$$

and the elimination of the parameters $\mu, \bar{z}, \gamma_{3}, \cdots, \gamma_{n}$ between the equations (9) and (10) will give the most general equation of order $n$ belonging to the class $(\alpha)$. But the preceding elimination can be performed without any modification if we start from

$$
\begin{aligned}
y & =\phi\left(z, \gamma_{0} \bar{z}+\gamma_{1} z+\gamma_{2}, \gamma_{3}, \gamma_{4}, \cdots, \gamma_{n}\right), \\
y^{\prime} & =\frac{\partial \phi}{\partial z}+\mu_{1} \frac{\partial \phi}{\partial k} ; \cdots ; y^{(n)}=\left(\frac{\partial}{\partial z}+\mu_{1} \frac{\partial}{\partial k}\right)^{(n)} \phi,
\end{aligned}
$$

where $k=\gamma_{0} \bar{z}+\gamma_{1} z+\gamma_{2}$ and $\mu_{1}=\gamma_{1}+\gamma_{0} \mu$.

* See E. Kasner, Science, vol. 66 (1927), pp. 581-582; Proceedings of the National Academy of Sciences, vol. 13 (1928), pp. 75-82; E. R. Hedrick, ibid., vol. 14. 
Let us denote by $E_{1}$ all the above eliminations. If $\gamma_{0}$ is replaced by zero in (11), and if we form the differential equation whose general integral is the monogenic function thus obtained, we get

$$
\begin{aligned}
Y & =\phi\left(z, \gamma_{1} z+\gamma_{2}, \gamma_{3}, \cdots, \gamma_{n}\right), \\
\frac{d Y}{d z} & =\frac{\partial \phi}{\partial z}+\gamma_{1} \frac{\partial \phi}{\partial k_{1}} \\
\ldots & \ldots \ldots \\
\frac{d^{n} Y}{d z^{n}} & =\left(\frac{\partial}{\partial z}+\gamma_{1} \frac{\partial}{\partial k_{1}}\right)^{(n)},
\end{aligned}
$$

where $k_{1}=\gamma_{1} z+\gamma_{2}$.

It is obvious that the elimination of $\gamma_{1}, k_{1}, \gamma_{3}, \gamma_{4}, \cdots, \gamma_{n}$ between (13) and (14), which elimination we will call $E_{2}$, will give the same result as $E_{1}$. Therefore any equation of the $n$th order belonging to the class $(\alpha)$ has a monogenic integral of the form

$$
Y(z)=\phi\left(z, \gamma_{1} z+\gamma_{2}, \gamma_{3}, \cdots, \gamma_{n}\right) .
$$

The converse is also true; if a differential equation has this property, it belongs to the class $(\alpha)$. Indeed, if (15) is its monogenic integral, let us form (11) by adding $\gamma_{0} \bar{z}$ to $\gamma_{1} z+\gamma_{2}$. The eliminations $E_{1}$ and $E_{2}$ giving always the same result, it follows that (11) is the polygenic integral of the same equation.

Hence, the property which determines the class $(\alpha)$ is that its monogenic integral contains in a special way two arbitrary constants.

It is obvious that (11) gives the most general polygenic integral of the equation of the $n$th order belonging to $(\alpha)$. Because, if $y(z)$ contains more than $n+1$ distinct arbitrary constants, their elimination could not give an equation of the $n$th order. Therefore, (11) is the form of the general polygenic integral of the equation of the $n$th order of $(\alpha)$. It contains $n+1$ arbitrary constants; the solution of Cauchy is a particular case obtained for $\gamma_{0}=0$. Inversely, if an equation of the class $(\alpha)$ is given, and if its monogenic general integral is known, the polygenic integral is obtained by adding the term $\gamma_{0} \bar{z}$ to $\gamma_{1} z+\gamma_{2}$.

3. In the preceding theory we have used rectilinear derivations; that is, we made $d z_{\theta}$ tend to zero in the expression for the higher derivatives

$$
\frac{d^{n} y}{d z_{0}^{n}}=y^{(n)}=\left(\frac{\partial}{\partial z}+e^{-2 i \theta} \frac{\partial}{\partial \bar{z}}\right)^{(n)} y,
$$

the affix of $d z_{\theta}$ lying on the straight line which passes through $z$ and makes the 
angle $\theta$ with $O x$. But we can generalize this conception by considering, as did Kasner, ${ }^{*}$ the "curvilinear" derivatives where the path of approach is any curve. It may be interesting to see which are the classes of differential equations which admit polygenic integrals, when we take the derivatives of $y$ in the general conception of Kasner, taking into account the curvature $\kappa$, and even other differential elements of a higher order, of the path curve on which $z+d z_{\theta}$ tends to $z$, this path curve being considered as entirely arbitrary.

It is obvious that the types thus obtained all belong to class $(\alpha)$.

* See his paper The second derivative of |a polygenic function, these Transactions, vol. 30 (1928), pp. 803-818.

UNIVERSTTY OF BUCHAREST,

Bucharest, Roumania 